\title{
Intention awareness: improving upon situation awareness in human-centric environments
}

\author{
Newton Howard ${ }^{*}$ and Erik Cambria
}

* Correspondence: nhmit@mit.edu Massachusetts Institute of

Technology, Cambridge, MA 02139, USA

\begin{abstract}
As the gap between human and machine shrinks, it becomes increasingly important to develop computer systems that incorporate or enhance existing Situation Awareness. However, these tend to focus on raw quantitative parameters, such as position and speed of objects. When these situations are governed by human actors, such parameters leave significant margins of uncertainty. In this paper, we discuss the potential of applying the characteristics intrinsic to the human actors that comprise a given situation to Situation Awareness, and the capacity that these concepts have to improve situation-aware systems. We argue that intention-aware based systems offer an advantage over situation-aware based systems in that they reduce the informational burden on humans without limiting effectiveness. We argue that computational analysis and tracking of semantic and affective information associated with human actors' intentions are an effective way to minimize miscommunication and uncertainty, particularly in time-sensitive and informationsaturated situations.
\end{abstract}

Keywords: Intention awareness, Situation awareness, Sentic computing

\section{Introduction}

As computer systems continue to improve in their information processing capability and become more integrated into the everyday lives of end-users, the potential scope of their roles increases as well. For instance, recent attempts to augment the human decision-making process, especially in dynamic and time-sensitive scenarios such as military command and control, game theory, home automation, and swarm robotics, have focused primarily on environmental details such as positions, orientations, and other characteristics of objects and actors of an operating environment. However, a significant factor in such environments is the intentions of the actors involved. While creating systems that can shoulder a greater portion of this decision-making burden is a computationally intensive task, performance advances in modern computer hardware bring us closer to this goal.

This paper discusses Intention Awareness (IA) as the process of integrating actors' intentions into a unified view of the surrounding environment. IA includes many of the basic principles of Situation Awareness (SA), such as consistent tracking and extrapolation of objects in the user's environment, but also exploits circumstantial semantics and sentics [1], that is, the conceptual and affective information associated

(C) 2013 Howard and Cambria; licensee BioMed Central Ltd. This is an Open Access article distributed under the terms of the Creative Commons Attribution License (http://creativecommons.org/licenses/by/2.0), which permits unrestricted use, distribution, and reproduction in any medium, provided the original work is properly cited. 
with objects and actors of the operating environment. Consider the everyday example discussed below.

Everyday tasks, from handling fragile objects to navigating a highway to parking one's car at work, require a high degree of SA and spatial aptitude. Such tasks require the human actor to quickly adapt to new stationary and moving objects, as well as unpredictable moves that these may make, such as a pedestrian suddenly crossing the road. In the case of parking a car, the driver must account for the overall distribution of objects in the space of the parking lot at the time he/she plans to park, as well as the actions those objects may take based on the intentions of other human actors, hence the Parking Dilemma [2]. In order to properly understand and predict the actions of others in this space, the driver must predict what others will do, or their actions will otherwise appear random. For instance, a car may suddenly change course to move to a parking spot, which a driver considering the intentions of other drivers is more likely to detect and account for in his subsequent driving maneuvers.

While cars and other motor vehicles possess functions, such as turn signals, to assist in the conveyance of the driver's intention to other drivers, there still remains a significant gap between a driver's intentions and other drivers' awareness of them. Due to finite time and resources, routine activities such as this require integrating not only SA, but IA as well, in order to optimize the exchange of those resources for some other reward (in the above example, a parking space).

In this paper, we discuss and deconstruct the SA paradigm from four distinct perspectives. First, we review relevant theoretical work in SA and organizational theory fields in order to assess their strengths, weaknesses, and their alternatives. We apply the results of this assessment in the second section, where we provide a working definition of SA, and in the third section, we demonstrate the additional utility that IA offers in enhancing the predictive analytical capabilities of SA. In the fourth and final section we summarize these findings and point to specific areas that would benefit from future development and integration of IA.

SA has grown to include a large scope of environmental and informational attributes, which depend primarily on the nature of the situation. In a maritime navigational scenario, for example, the situational picture would include other ships, weather, wind, depth, buoy locations and heading. On the other hand, the parking scenario, which generally entails more direct association with other actors over limited resources, would include a greater emphasis on other driver's intentions. Thus, employing SA properly requires a significant devotion of cognitive (and, more recently, computational) resources as well as recognition of the appropriate level of focus for new input and ongoing analysis. That is, staying in the bounds of a sea channel takes on a lower priority in an emergency collision avoidance maneuver at sea.

In order to achieve this level of focus effectively, both human actors and their computer system counterparts need to be able to analyze the situation both with existing data and knowledge about other actors' goals. Data-driven analysis is based on tracking the status of existing goals as new data about the situation become available, and goaldriven analysis is the basis for the formation of new goals (if necessary) based on the results of data-driven analysis. Because each mode of analysis depends on the other, the situation-aware entity, human, or computer, must be able to switch dynamically between them. 
Because warfare condenses and renders more urgency to the requirements of SA, many of its original concepts can be traced to scholarship on military operations, such as Clausewitz's writings on the "frictions" of war, one of the most important being uncertainty, or the "fog of war [3]." Apart from warfare, SA's innovations have proven useful in all scenarios, including any in which high-stakes, short-term variations are present.

As a result, study of aviation and air traffic control has also yielded significant insight into augmenting human SA [4]. This has resulted in a proliferation of definitions for SA, some more domain-specific than others. However, the most cited definition is that provided by Mica Endsley in 1988:

The perception of the elements in the environment within a volume of space and time, the comprehension of their meaning, the projection of their status into the near future, and the prediction of how various actions will affect the fulfillment of one's goals [5].

In this passage, Endsley is describing the critical process of situation assessment. Situation assessment is a combination of information acquisition and interpretation, which consists of four distinct but integrated stages:

1. Perception: acquiring the available facts.

2. Comprehension: understanding the facts in relation to our own knowledge of such situations.

3. Projection: envisioning how the situation is likely to develop in the future, provided it is not acted upon by any outside force.

4. Prediction: evaluating how outside forces may act upon the situation to affect our projections [Ibid].

Vidulich et al. [6] provide some elaboration on Endsley's previous definition describing SA as "the continuous extraction of environmental information, the integration of this information with previous knowledge to form a coherent mental picture, and the use of that picture in directing further perception and anticipating future events". Both Endsley and Viludich appear to agree that SA, which occurs in the present, has the primary goal of forming a coherent narrative between past, ongoing events, and likely future outcomes. While each of their definitions is technically compatible with the IA concept, the lack of implementation of IA at the time they published their theories on $\mathrm{SA}$, and in the present to some degree, has been the primary cause of the conceptual gap between IA and SA.

As an outcome of situation assessment, SA can be viewed as a quadripartite end product of an actor's existence in a given scenario. Since SA occurs in four critical stages (per Endsley's definition), they can each be mapped to their own "level" in the SA hierarchy. Perception corresponds to level 1 SA, comprehension to level 2, and projection to level 3, respectively. Demonstrating the utility of this perspective, Jones and Endsley [5] were able to isolate $76 \%$ of SA failure in pilots could as problems in level 1 SA (perception of needed information) due to failures and/or shortcomings in the system or in cognitive processes themselves. The study also found that $20 \%$ of SA failures were attributable to level 2 SA (projection), and the remaining $4 \%$ involve problems with level 3 (projection). 
While this study demonstrates a rough analytical approach to intentions and perceptions, it also shows the necessity of a more precise understanding of the interaction between intentions of human actors and the situations in which they find themselves. Endsley and Jones demonstrated significant correlation between these phenomena in their study, suggesting a need for further study. Of particular importance is the question of whether the limits of the human brain's capabilities impose a limit on computationally augmented SA. If so, a more cognitive-based approach to the SA problem is warranted.

Situation assessment is an intensive cognitive process that serves as a junction between the physical properties of objects (position, heading, etc.) and the human mind's portrayal of such objects. In order to successfully complete this process, the actor conducting situational assessment must be able to properly account for the intentions of the other actors that share the same situation. This is a shortcoming in many contemporary SA models, which give less priority to intentionality as a driver of human-dominant situations [2,5,7]. Due to the inherent structural differences in situational data and intentional information, not all systems that address the latter can also address the former, which produces a significant implementation gap despite the conceptual and potential application overlap between SA and IA.

It is also known that attention plays a significant part in successful SA [8-10]. Up to 35\% of all SA failures in Endsley's study were traceable to attention; "all needed information was present, but not attended by the operator" [5]. Although it may appear to be explicitly tied to perception, attention is also affected by intentions and goals. "[A] gents deployed their attention in ways that are consistent with [their] operational goals” [4].

Goals are important in cognitive models in various other ways as well:

a) "active goals direct the selection of the mental model,"

b) "goal and its associated mental model are used to direct attention in selecting information from the environment,"

c) "goals and their associated mental models are used to interpret and integrate the information" [11].

Each of these precepts is representative of a classical interpretation of intentions in which cognitive states constantly "evolve" into hierarchical forms based on the demands of the environment.

Bruner and several other scholars of cognition and SA suggested that goal-driven planning is a subconscious cognitive process. Thus, the action resulting from this process can be viewed as hierarchical in nature [ibid]. Similarly, intention itself is comprised of several discrete layers, each having a unique relationship to consciousness; there are high-, medium-, and low-level intentions: "A hierarchy is formed by these three levels of intention that give us a relation between the means and ends" [12].

High-level intentions are the behaviors, as well as the beliefs and emotions that drive them, that one actor will use to influence another actor. Mid-level intentions are related to high-level intentions in that they are used to achieve the goals defined by high-level intentions, and low-level intentions provide a means to achieve goals defined by intentions at either of the higher levels. This hierarchy serves to simplify spatio-temporal reality by reducing it to states and actions. 
In very general terms, we can describe all human activities as a series of proactive and reactive states and behaviors that cause transitions between states. Actors themselves have internal states, i.e., "states of mind," and they use these mind states to perceive the interactive environment they inhabit. Because human beings are themselves cognitive systems, and operate in a greater environment that contains other humans, any given situation can be described as a system of systems. Humans in this system are inherently intentional, exhibiting certain consistently rational behavior based on their intentions [13].

When we refer to rationality within this paper, we are specifically discussing "acting as best to satisfy [one's] goals overall, given what [one's] know and can tell about [one's] situation" [14]. In this sense "rationality is the mother of intention" [ibid]. According to this definition, intention can be described as a relationship between some object (primarily physical such as an artifact) and an actor's internal mind state - desire, belief, goal, purpose, etc. Intentionality ${ }^{\mathrm{a}}$ is thus "the character of one thing being "of" or "about" something else, for instance by representing it, describing it, refereeing to it, aiming at it, and so on" [ibid].

At the lowest level, we can view intention as the relationship between an environmental state and the objects with which it interacts and which act upon it. Intentional stance is an abstract tool that enables the predictive analysis of complex systems such as human actors, but does not account for the cognitive nature of those processes and the way that they operate [15].

If an intentional stance exists in a system by virtue of human presence, this takes us to the next phase in the process - identifying the system state and its components. Such a system can be characterized by myriad factors, including but not limited to beliefs, goals, wants, previous commitments, fears, and hopes. States within the system can be viewed as "attitudes" and grouped as such. Below is an example of one such grouping:

1. Information attitudes: preconceived notions that an actor has towards information about the surrounding environment.

2. Proactive attitudes: attitudes that direct a mind state to favor action.

3. Normative attitudes: obligations and permissions [ibid].

Prior research has, for the most part, followed a work template that concentrates primarily on one of these attitudes and/or notions and investigates others in relation to it. Research investigating the relationships among beliefs, desires, and intentions (BDI) assumes that these systems project inherently desired states (and that the nature of these desired states does not change with the environment) [16].

While BDI and classification systems for attitudes present researchers with a straightforward abstraction of environmental systems, there are a number of alternative, abstract views for the analysis of interacting systems such as human actors situated in a given environment. These types of systems can be regarded as exceptional cases, or intentional systems, because they involve the interaction of human actors and thus depend heavily on modeling intentionality. If the human actor must choose how to act in both a spatial and temporal frame, then we can model the system and its inputs by addressing the factors that contribute to those choices. In these systems, observers often note a consistent, rational behavior pattern that can be mapped to intuitive intentions on the part of the human actors in the system [14]. 
Intentional systems differ from conventional ones in that they possess several additional explanatory characteristics and capacities. First, they tend to view infrequent conditions as similar to more frequent ones because classification depends more on relevance to intentions than empirical frequency. This gives intentional systems the ability to create and analyze "multidimensional" states. This allows a simple, straightforward means to solve the problem of scaling and context.

We can thus construct a useful abstraction for giving a general description of systems that will extend past specificity of individual attitudes to include their interaction with other systems, even those that do not specifically include human actors, such as organizations and computer systems. To do so, we must consider multiple levels of abstraction based on the following criteria:

1. Intentional states. These must be defined in accordance with the relationship between states and relevant objects, if the system in question is intentional. The analysis must also distinguish between desires, beliefs, and intentions because these are fundamentally distinct intentional states.

2. Types of choices. Choices are defined based on the characteristics of interpretation, justification, and functionality of specific behavioral decisions. For instance, justifiable choices by some set of observers, primary or secondary, qualify for classifying choices - these qualifications are often made based on the mechanism by which those choices are made.

We can also specify two schemes, or classes, to simplify the definition of choice types in system characterization. The first defines the various types of intentional states, and the second defines types of choices to be made that will ultimately affect those states. Similarly, intention schemes (I-schemes) and choice, or rationality, schemes (R-schemes) can be used to map intentions to their logical behavioral conclusion.

All systems that include some degree of intentionality can thus be analyzed as instances of intentional systems with different levels of I-schemes and R-schemes. Systems composed of animal actors are equipped with this intentionality and rationality, but in a way that is distinct from their expression in human agents. In addition to humans, information systems such as computers and computer networks possess a similar rationality/intentionality scheme. Specifically, their behavior is guided by transitions from initial states to non-initial states by way of intrinsically motivated behavior, or intervention from without.

\section{Distributing SA among human actors}

Artman et al. [17] argue that systems driven by teams of actors need an additional dimension of analysis: "it is necessary to shift the unit of analysis from the individual to the whole cognitive system comprising a team of people as well as the artefacts which they use." This is due to the fact that, while examples of SA are often single-user centric, team-driven action multiplies the complexity of the SA picture by the number of actors involved, because the difference between each of their perceptions will create distinct outcomes. Thus, Artman et al. touch on a SA concept that is not entirely unrelated to IA, which is the multiplicity of actors. The primary distinction is that in IA, we address all actors that impact the environment, not just those that form a loose unit that functions as a larger actor (Figure 1). 


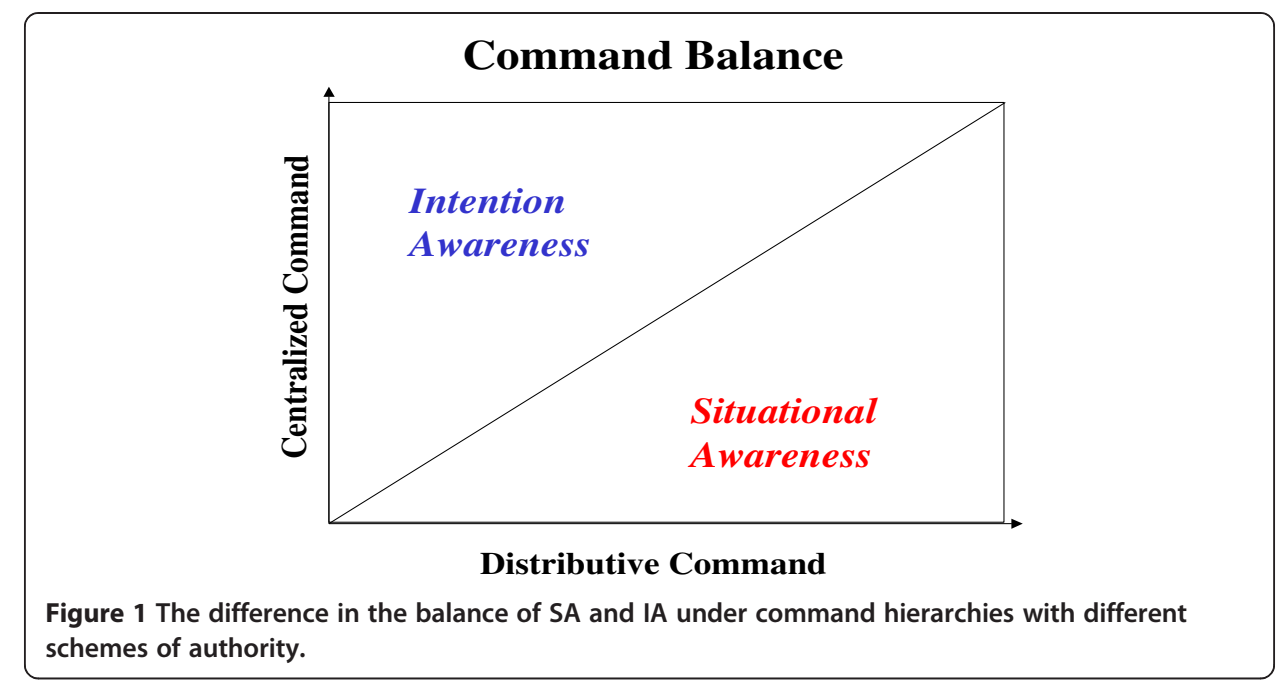

\section{Intention awareness}

IA is the process of integrating actors' intentions into a unified view of the surrounding environment. IA includes many of the basic principles of SA, such as consistent tracking and extrapolation of objects in the user's environment, but also exploits circumstantial semantics and sentics associated with objects and actors of the operating environment. Semantics and sentics, in fact, are key for sensemaking, in which they extend the meaningfulness that the system attributes to every single object/actor of the environment by considering it as an entity carrying specific conceptual and affective information, rather than simply a point in a space or time window. In the context of home automation, for example, a greater deal of intentions can be inferred, if the semantics and sentics associated with the object 'coffee machine' are known. Such semantics and sentics can be retrieved from external resources, e.g., affective common-sense knowledge bases for concept-level sentiment analysis [18]. For example, if the system has access to pieces of information such as 'a coffee machine is used for making coffee' and 'people generally like coffee', the repeated proximity of a human actor to the object 'coffee machine' can be exploited to infer the intention of the human actor to make coffee.

\section{Intention in computation}

The BDI model functions by establishing a decision tree whose nodes represent possible future outcomes, and assigning probabilities to each of these based on calculations of intent. Computational models of intention, such as the BDI software model, attempt to optimize the time spent allocating computational power for given tasks and actually executing them. While the BDI model is fairly rudimentary in its analysis of intent (essentially parsing past requests and actions to find trends), it epitomizes the increasing feasibility of implementing IA in computational systems. Four basic components of BDI allow speedy processing of intentional information:

- Beliefs

- Desires

- Intentions

- Events 
BDI formal architecture consists of "beliefs," or the set of inference rules that an agent uses to derive basic intentional information. "Desires" represent the distinction between goal end-states and the current state. That is, desires are tasks actively pursued by the agent, such as performing a mathematical operation on a set of numbers. Intentions in BDI represent what has already been decided upon by the agent, but is not necessarily being achieved yet. For instance, the primary distinction between intentions and desires is the level of commitment. Intention, both in human and computational systems, requires some sort of signaling to transform from desire.

What is particularly significant about BDI modeling in software is its use of the intent/action chronological dichotomy to construct information-rich event narratives. While more mature implementations of intention in computational systems will be inherently intention-aware than BDI, this model illustrates the importance of actordriven decision making.

\section{Formal models of intention}

In the Control Theory approach to system behavior analysis, states, or the distinction between intentions and whether they have been executed and goals, or short-term components of intention, are central. For instance, feedback control mechanisms are components of systems constructed for the purpose of reducing the difference between an actor's goal and their current state, and these systems tend to exhibit some form of efficiency or consistency, such as the consistent rational behavior we attribute to intentionality in humans [19-21]. The important distinction between goal and present systemic state is traceable to the type of applied rationality, i.e., in selecting strategies. "Feedback control reacts to sensory observations with actions designed to correct errors between observed and desired states," while "feed-forward uses a model of the world to predict what actions are required to achieve the goal" [22].

The distinguishing characteristic of feedback control is that is data-driven, and often results in reactive behavior. Observers can obtain information about past events, but such definitive information is largely unavailable when future events, beliefs, and perceptions are under analysis and, hence, projection plays a significant role in perception. Thus, feed-forward control results in proactive anticipatory behavior.

Control over a system is, simply put, a means of reducing the variety that the operating environment imposes. In a military environment, superior intelligence offers battlefield commanders greater control because this necessarily reduces variety (i.e., uncertainty). Ashby's Law of Requisite Variety tells us that "only variety in [response] can force down the variety due to [disturbance]; variety can destroy variety" [23]. Simply put, a system reacting to variety in the environment must have more inherent variety in order to seek its goals.

Reactive response in terms of possibly unlimited variety creates a unique problem. Specifically, the system's internal intrinsic variety must be maximized so that unexpected developments can be dealt without losing control. The best way to solve a problem, in fact, is to already know a solution for it. But, if we have to face a problem we have never met before, we need to use our intuition. Intuition can be explained as the process of making analogies between the current situation and the ones solved in the past to make a suitable decision at the present time. Such reasoning by analogy can be emulated, by means of sentic computing [1], through the ensemble application of semantic multi-dimensional scaling [24] and neural-network-based clustering [25]. 
Feed-forward controls assume that systems are reflective of the environment in which they are situated and that they can also predict states and actions by analysis of that system. As Ashby predicts, "Any regulator able to confine the fluctuations in the system to be regulated must not only have adequate amounts of variety available to control that system but also be or have a homomorphic representation of that system" [26]. In the early 1970s, Maturana and Varela developed the theory of Autopoiesis, which tends to support this perspective [27,28]. Autopoiesis is closely related to self-referentiality, or self-awareness attributes that we associate with human actors, and sees living systems as dualistic. That is, Autopoiesis covers both the internal organization of these systems, such as structure and metabolic functions, as well as their external component, or agency. The theory does this by presenting three key notions: operational closure, component production networks (which is an abstraction of the concept of metabolism), and spatio-topological unity between the individual and any physical borders he/she may encounter. Proponents of Autopoiesis claim that recursivity, or an organization's resemblance to a closed loop, is generated by the components and production processes inherent to the system that develops a complementary relationship between the network and border $[2,28]$. This system begins to develop a unique identity based on the set of couplings and shared components and processes within it, and thus a holistic character of its component processes $[27,29]$. Such a system must also be able to regulate the flow and consumption of matter and energy in order to facilitate self-constructive processes, as well as exchanges of material and information with the surrounding environment. The system must therefore be able to generate its defining factors, such as boundary conditions, that define it as such.

Based on this discussion, we propose a list of necessary attributes for systems incorporating intentionality in any way:

1. Self-existence is necessary because this will allow the system to increase its internal variety consistently.

2. The Rule of Requisite Variety must be satisfied in s series of transitory states at least once, which leads to the formation of intention.

3. We can also deduce that since intentions are hierarchical, they are so organized according to their inherent rationality. Thus, a system will exhibit more unconventional behavioral solutions to problems presented by the environment by forming intentions of its own volition, and then acting upon them. This is also observed in studies of AI and intentionality, such as in [15] citing [30] "that Sandy [,] of the Coffee shop Conversation [,] claims that the really interesting things in AI will only begin to happen when the program itself adopts the intentional stance towards itself."

4. Intentional systems with higher internal variety tend to reach their goals more frequently in constantly changing environments.

5. The internal state of a system tends to be richer in terms of intentionality and rationality than its external state.

6. Competing instances of rationality and intentionality contribute to an operating environment's current state, and state transitions can be attributed to the result of these phenomena. This type of competition helps to explain why the potential 
internal variety of an individual system is greater than the actual scope of states that an operating system can exhibit.

7. Individual system intentionality can be independent, shared, complementary, and conflicting.

8. Because intentionality is hierarchical, there are equivalent mappings between actions and intentions.

9. Intentionality is shaped not only by semantics associated with the operating environment, but also by sentics each individual system associates with specific objects and events of that environment.

A number of scholars argue that goal-directed planning happens below the threshold of consciousness and that as a result, components of action are organized in a more hierarchical fashion [12,31]. Affective information processing, in fact, mainly takes place at unconscious level [32]. Reasoning, at this level, relies on experience and intuition, which allow considering issues intuitively and effortlessly through reasoning by analogy in a multi-dimensional space where the exact relationships between specific concepts are lost, but in which it is easier to infer how such concepts are semantically and affectively related [33]. Hence, rather than reflecting upon various considerations in sequence, the unconscious level forms a global impression of the different issues. In addition, rather than applying logical rules or symbolic codes (e.g., words or numbers), the unconscious level considers vivid representations of objects or events. Such representations are laden with the emotions, details, features, and sensations that correspond to objects and events of the operating environment.

Forming a hierarchy of high-, medium-, and low-level intentions presupposes that the intentional system in question possesses a highly nuanced comprehension of the operating environment in which other individuals' intentions are formed, as well as relevant details about the other individuals themselves.

We employ Lattice Theory to bring some insight to this complex problem of intentions, actions, and attributes. The Law of Modularity is of particular use here, since it tells us that for any three components of a lattice $a, b$ an $c, x \leq b \rightarrow \rightarrow x \vee(a \wedge b)=(x \vee a) \wedge b$, using an AND operation to represent the joining operation, and the OR operation to portray the meet operations. What this tells us is that for a modular lattice, the highest lower bound on a partial order within the lattice is inversely isomorphic with the lowest upper bound.

We can then apply the Law of Modularity to our intentional hierarchy because in order to construct intentions, they must first be isomorphic because they influence each other in some way, no matter how trivial. For instance, we already know that the development of higher-level intentions gives rise to low- and middle-level intentions, and that high-level intentions can only be expressed through some combination of these. Interactions between any of these types of intentions are necessarily isomorphic, similar to those between high-level intentions, because they all exist within the same set of physical parameters. As a result, some interaction between subsets of intentions and actor systems will lead to unexpected results at lower levels. Representing intentions as lattice elements allows a better understanding of the intentional environment's inherent structure, as well as a better understanding of the interactions among them. 
Using the lattice structures, we can represent these interactions between sets of intentions as follows:

1. Let the set of all of an individual's intentions be $S$, where $\{S:(l \cup m), h\}$.

a. $l$ is the set of low-level intentions;

b. $\mathrm{m}$ is the set of mid-level intentions;

c. $h$ is the set of high-level intentions.

2. If $\{l, m, h\} \subseteq S, \forall i \in(l \cup m), \exists j \in h$ such that we can define a mutually isomorphic relationship between $i$ and $j$.

Assigning new information the proper value and priority in a rapidly changing environment remains a challenge to engineers and scholars of SA, since it is not simply the ability to acquire new information, but to interpret it in a way that is beneficial to the actor's intentions [34]. Here quality trumps quantity; that is, information must be useful more than it must be plentiful. As a simple example, imagine three email servers: one that filters no junk email, one that occasionally marks an important message as junk, and one that occasionally lets junk mail into the inbox. The third server is clearly the superior application of SA, since it prevents inundation (albeit imperfectly) without sacrificing the ultimate mission of the email server, which is to convey important information.

\section{Applying psychological models of intent}

In her research into human intentions, Zeigarnik argued that the "effect of intention is equivalent to the creation of an inner personal tension," meaning that one system is in disequilibrium of relative to surrounding systems. This relative equilibrium is a manifestation of the fundamental forces that cause equalization of other systems. This conclusion suggests that the human mind is itself a system with its own dynamic forces and subsystems. Zeigarnik made four basic assumptions in his theory [31]:

Assumption 1: The intention to reach a certain goal $G$ (to carry out an action leading to $G)$ corresponds to a tension $(t)$ in a certain system $S(G)$ within the person so that $t(S(G))>0$. This assumption coordinates a dynamic model (system in tension) with "intention."

Assumption 2: The tension system $t(S(G))$ is released if the goal $G$ is reached. $T(S(G))=$ if $P$ accomplishes $G$. Zeigarnik uses the tendency to recall activities as a symptom for the existence of tension The expectation of the existence of such a system is based on the following:

Assumption 3: To a need for $G$ corresponds a force $f(P, G)$ action upon the person and causing a tendency of locomotion toward $G$. If $t(S(G))>0$ then $f(P, G)>0$. This assumption determines the relation between need and locomotion - motion from one place to another place. In other words it means a construct of tension in the person and the construct of force for locomotion in the environment.

Assumption 3a: A need leads not only to a tendency of actual locomotion towards the goal region but also to thinking about this type of activity; in other words, the force $f$ 
$(P, G)$ exits not only on the level of doing (reality) but also on the level of thinking (reality); if $t(S(G))>0 f(P, R)>0$ where $R$ means recall.

From these assumptions we can theorize that intentional systems such as humans have a stronger tendency to recall interrupted activities than finished ones. We can make the corresponding derivation as follows, where $\mathrm{C}$ is the completed task, $\mathrm{U}$ is the unfinished task, and the corresponding systems are represented by $\mathrm{S}(\mathrm{C})$ and $\mathrm{S}(\mathrm{U})$, respectively [2]:

1. $\mathrm{t}(\mathrm{S}(\mathrm{U}))>0$ according to Assumption 1

2. $\mathrm{t}(\mathrm{S}(\mathrm{C}))=0$ according to Assumption 2

3. $\mathrm{f}(\mathrm{P}, \mathrm{U})>\mathrm{f}(\mathrm{P}, \mathrm{C})$ according to Assumption $3 \mathrm{a}$

Note: Zeigarnik computed the ratio as $R U / R C=1.9$, where $R U$ is unfinished tasks, an $R C$ represents completed tasks.

This model has several prerequisites regarding the dynamic character of this field, specifically with respect to the maintenance of tension and internal variety during a specific period of time. Since we expect this to be a very fluid field, any differences between the tension levels of the various systems will tend to disappear quickly as they tend toward equilibrium.

A human actor thus must have some degree of variance, or fluidity, in regard to the communication of his/her systems that cause tension. Thus type of fluidity must clearly vary between people and situations, but if we assume the constancy of structural relations between them, we can express them in the following way [2]:

Corollary: Let us indicate the absolute difference between the tension $t(S 1)$ and the tension $t(S 2)$ of two neighboring systems S1 and S2 at the time the tensions are being built up by the time since then elapsed by Ti, the tension difference at this time by, and the fluidity by. Then we can state, where symbolizes a monotonously increasing function. This means: the change in the tension difference of neighboring systems depends upon the time interval and the fluidity. Of course, this holds true only if the tensions of these systems are not changed by other factors such as, e.g., release of tension by reaching the goal?

Applying field theory to this new observation, we can make the following propositions:

a) Behavior is derived from the universe of coexisting facts;

b) These coexisting facts are similar in behavior to a dynamic field. That is, the state of any subfield depends on the rest of the field as a whole.

Proposition (a) presupposes we are dealing with a manifold, the internal relationships of which must use the concept of space for representation.

Spatial relationships between different psychological data cannot be represented in physical space, and instead must be treated as existing in mental space. It is everywhere accepted that the "life space" includes the person and his mental environment or world. 
Locating and applying an appropriate geometry that can represent the spatiotemporal relationships of psychological facts is one of the first requirements of representing the mental space. With modern computational hardware, this is more possible today than ever before, especially in Zeigarnik's era. Today, one can find many geometries that permit mathematical understanding of the hodological space.

This is a finitely structured space, meaning that its subspaces and components are not infinitely divisible, but they are composed of certain units. Direction and distance are quantities that can be represented by "distinguished paths," which are easily correlated to psychological locomotion.

The geometry of hodological space is sufficiently well-defined that it can adequately represent most psychological processes, and it also permits an answer to the puzzling necessity to ascribe different psychological intentions and actions to locomotions in the same physical direction and dimension. This feature is particularly critical for the roundabout route problem: hodological space permits the description of structural relations inherent to the actor as well as in psychological operating environment.

Hodological space is equally useful for describing the structure and functions of groups of people. It is even more useful, however, when describing dynamic systems. Field theory tells us that behavior depends on the present field, not either the past or future fields. However, this stands in contrast to the theory of teleology, which opines that the future is the cause of behavior, and associationism, which opines that the past is the cause of behavior.

In addition, one must not make the mistake of assuming that directed factors are in themselves characteristic of teleology. These causal assumptions are even visible in physics, since physical force is a directed entity consisting of magnitude and the directional quality that allows its representation as a vector. The same goes for metapsychology, which resorts to constructs of similar vector-like character, such as psychological forces.

By defining directed forces in hodological space, we can adequately represent other teleological claims. The strange relationship between knowledge and dynamics that teleology has attempted to represent intuitively is made more comprehensible in one fundamental way: it becomes clear why ignorance serves as a barrier.

Zeigarnik's model provides a useful basis for modeling IA. The A-type model is the present model of the world. The type B model is a hypothetical future model of the world based on intentions and their ability to be achieved. A system of intentions can be used to define a map from a type-A-model to type-B-model, and finally, there is SA of the type-A-model. The fact that each agent at a given point in time has two models (SA and IA) creates many challenges to highlight, including the proliferation of multiple perceptions, expectations and awareness models that sometimes come into conflict with one another.

Thus emerges the four-part hypothesis [2]:

1. Model $A(X)-$ Model $A^{\prime}(X) 0$.

- Agent $X$ type- $A$ model at time $t 1$ cannot be equal to Agent $X$ type-A model at time $t 2$.

2. Model $B(X)-$ Model $B^{\prime}(X) 0$.

- Agent $X$ type- $B$ model at time 11 cannot be equal to Agent $X$ type- $B$ model at time. 
3. Model $A(X)-$ Model $A(Y) O$.

- Agent $X$ type- $A$ model at time $t 1$ cannot be equal to Agent $Y$ type- $A$ model at time 2 .

4. Model $B(X)-$ Model $B(Y) 0$.

- Agent $X$ type-B model at time $t 1$ cannot be equal to Agent $Y$ type- $B$ model at time $t 2$.

\section{Intention awareness: a new role for intent in computation}

Linking intent to SA-driven pictures of the environment requires more than simply creating an "intent" category in data analysis. From a computational perspective, intentions are causal inferences made from a series of events and a series of prior, linked intentions. Thus, in order for a system to possess IA, it must not simply infer based on available (i.e., situational) data, but must incorporate prior intentional analysis as one of its information sources. Thus, one of the fundamental distinctions between SA as we now know it and IA is that, while the former concerns data analysis that humans probably cannot process in the amount of time required, the latter concerns information analysis, a task that humans perform on a regular basis, but that can be enhanced by artificial systems (Figure 2).

One promising example of the extension of intention into SA is computational "sensemaking," or the process by which humans (or other reasoners) attach semantics and sentics to their observations of the operating environment in a parallel and dynamic way [35]. IA, in fact, is a viable means of improving synchronization in sensemaking between the human reasoner and associate system interfaces. This is due to two primary factors. The first is that IA is becoming increasingly viable thanks to advances in computer hardware performance. Second, since IA simultaneously frees some of the analytical

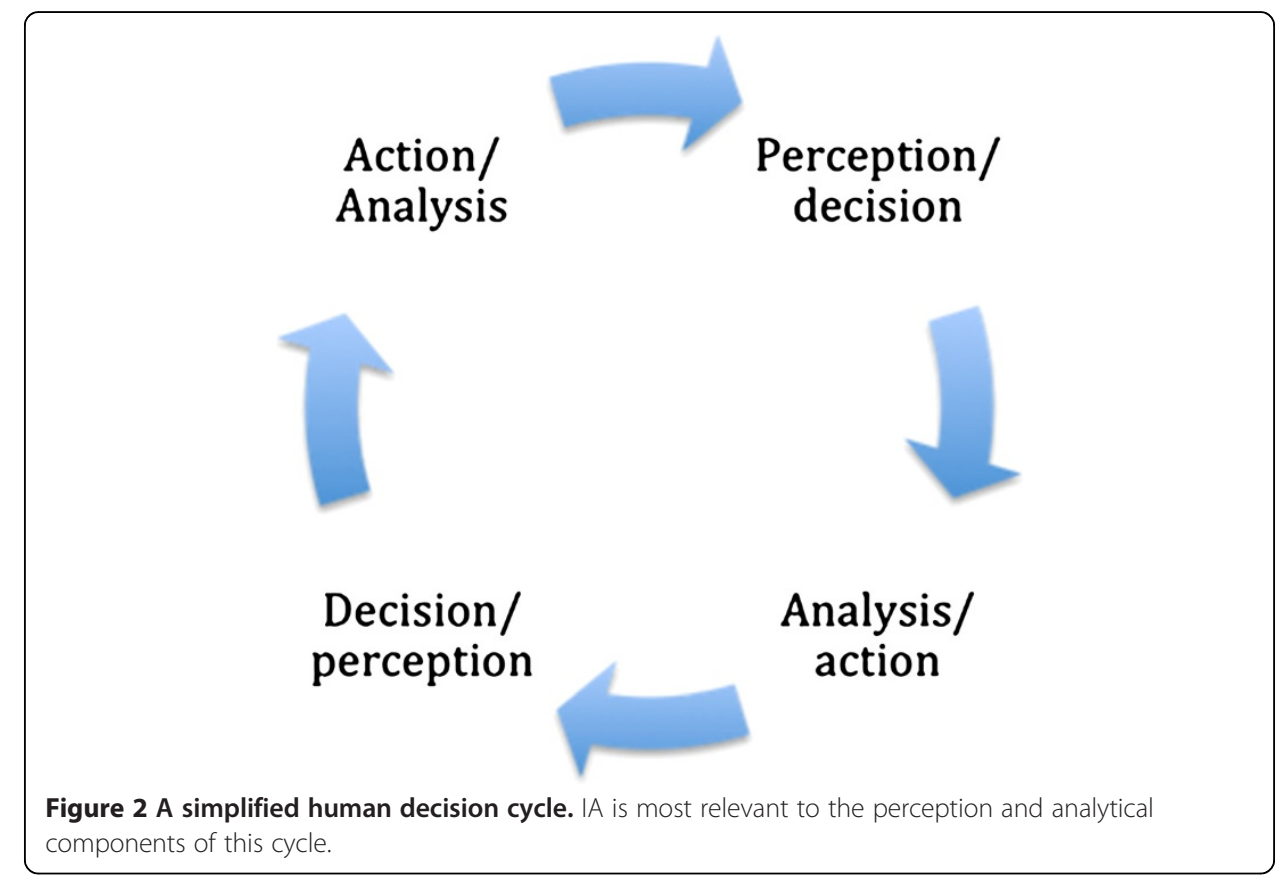


burden from human operators and uses similar structures as are found in human cognition, interface-based latency is minimized.

\section{Conclusion}

In popular applications, SA is seen as a proactive, purposeful behavior that is directed towards achieving a goal in a specific task environment. This view has been readily adopted in many different fields and contexts, where situations are by nature dynamic and subject to rapid, unexpected variation. Because commander's intent drives the mission, regardless of operational size or scope, it is tempting to cite military science as the primary beneficiary of advances in SA and IA systems. However, expansion upon some of the notions that drive the process of military organization and action reveals that we can apply the same concepts to any system requiring SA, e.g., game theory, home automation, cyber-security, and swarm robotics. This paper has argued that the next logical extension of SA is IA, or the independent analysis and awareness of intentions by computational systems that augment the human actor.

This is for two primary reasons. First, our understanding of cognitive processes has allowed a more computationally feasible problem of intent to be defined as the basis for IA models. Second, computational resources have made significant progress since the notion of SA was first proposed, so we currently experience an implementation gap where we have the resources but not the immediate capability to execute IA in field applications.

\section{Endnote}

${ }^{a}$ For the purposes of this paper, the concept of intentionality is the quality of having intentions, not to be confused with a quality of actions, as sometimes mentioned in the literature.

Competing interests

The authors declare that they have no competing interests.

\section{Authors' information}

Newton Howard (nhmit@mit.edu) is the director of the Synthetic Intelligence Lab at MIT and former Director of the Mind Machine Project. He received his Doctoral degree in Cognitive Informatics and Mathematics from La Sorbonne, France where he was also awarded the Habilitation a Diriger des Recherches for his leading work on the Physics of Cognition (PoC) and its applications to complex medical, economical, and security equilibriums. While a graduate member of the Faculty of Mathematical Sciences at the University of Oxford, England, he proposed the Theory of IA, which made a significant impact on the design of command and control systems and information exchange systems at tactical operational and strategic levels. He has served as the Chairman of the Center for Advanced Defense Studies (CADS), the leading Washington, D.C, National Security Group and is currently its board director. He is a national security advisor to several U.S. Government organizations. He recently published The Mood State Indicators (MSI) algorithm, which modeled and explained the mental processes involved in human speech and writing, to predict emotional states. Dr. Howard works with multi-disciplinary teams of physicists, chemists, biologists, brain scientists, computer scientists, and engineers to reach a deeper understanding of the brain. Dr. Howard's most recent work focuses on the development of functional brain and neuron interfacing abilities. To better understand the structure and character of this information transfer, he concentrated on theoretical mathematical models to represent the exchange of information inside the human brain. This work has proven applicable in the diagnosis and study of brain disorders and has aided in developing and implementing necessary pharmacological and therapeutic tools for physicians. He has also developed individualized strategies to incorporate solutions for psychiatric and brain prosthetics.

Erik Cambria (cambria@media.mit.edu) received his BEng and MEng with honors in Electronic Engineering from the University of Genoa, in 2005 and 2008 respectively. In 2011, he has been awarded his PhD in Computing Science and Mathematics, following the completion of an industrial Cooperative Awards in Science and Engineering (CASE) research project, which was born from the collaboration between the University of Stirling, Sitekit Solutions Ltd., and the MIT Media Laboratory, where he currently works as associate researcher (Synthetic Intelligence Project). His interests include Al, Semantic Web, KR, NLP, big social data analysis, affective and cognitive modeling, intention awareness, $\mathrm{HCl}$, and e-health. Dr. Cambria is invited speaker/tutor in many international venues, e.g., IEEE SSCI, MICAl, and 
WWW, associate editor of Springer Cognitive Computation, and guest editor of leading Al journals, e.g., IEEE Computational Intelligence Magazine, Elsevier Neural Networks, and IEEE Intelligent Systems. He is also chair of several international conferences, e.g., Brain Inspired Cognitive Systems (BICS) and Extreme Learning Machines (ELM), and workshop series, e.g. ICDM SENTIRE, KDD WISDOM, and WWW MABSDA.

\section{Authors' contributions}

$\mathrm{NH}$ developed the Intention Awareness in human centric environments concepts, and analyses. EC applied IA theory in Sentic environments. Both authors wrote, read and approved the final manuscript.

\section{Acknowledgments}

The authors would like to thank lan Andrews, Rebecca Fahlstrom Sergey Kanareykin, David Johnson, Ammar Qusaibaty, and Adam Howard for their contribution to the ideas discussed in this paper and their assistance in reviewing and refining this article.

Received: 27 September 2012 Accepted: 28 May 2013

Published: 12 June 2013

\section{References}

1. Cambria E, Hussain A (2012) Sentic Computing: Techniques, Tools, and Applications. ISBN: 978-94-007-5069-2. Springer, Dordrecht, Netherlands

2. Howard N (2002) Theory of Intention Awareness in Tactical Military Intelligence: Reducing Uncertainty by Understanding the Cognitive Architecture of Intentions. Author House First Books Library, Bloomington, Indiana

3. Spick M (1998) The ace factor: Air combat and the role of situation awareness. Naval Institute Press, Annapolis, MD

4. Endsley MR (2000) Theoretical underpinnings of situation awareness: A critical review. In: Endsley MR, Garland DJ (eds) Situation Awareness Analysis and Measurement. Lawrence Erlbaum Associates, Inc Publishers, Mahwah, NJ, pp 3-32

5. Jones DG, Endsley MR (1996) Sources of situation awareness errors in aviation. Aviat Space Environ Med 67(6):507-512

6. Vidulich M, Dominguez C, Vogel E, McMillan G (1994) Situation awareness: Papers and annotated bibliography, Armstrong Laboratory, Crew Systems Directorate, Human Engineering Division, Wright-Patterson, Interim Report for Period 15 January 1992 to 6 June 1994. AFB OH, AL/CF-TR-1994-0085

7. Lewin K, Cartwright D (eds) (1951) Field Theory in Social Science: Selected Theoretical Papers. Harpers, Oxford, England

8. Endsley MR, Smith RP (1996) Attention distribution and decision making in tactical air combat. Hum Factors 38(2):232-249

9. Endsley MR, Rodgers MD (1998) Distribution of attention, situation awareness, and workload in a passive air traffic control task: Implications for operational errors and automation. Air Traffic Control Quarterly 6(1):21-44

10. Gugerty L (1998) Evidence from a partial report task for forgetting in dynamic spatial memory. Hum Factors 40(3):498-508

11. Endsley MR (1995) Toward a theory of situation awareness in dynamic systems. Hum Factors 37(1):32-64

12. Gibbs RW (2001) Intentions as emergent products of social interactions. In: Malle BF, Moses LJ, Bladwin DA (eds) Intentions and Intentionality. MIT Press, Cambridge, MA

13. Dennett DC (1987) The Intentional Stance. MIT Press: A Bradford Book, Cambridge, MA

14. Haugeland J (1997) What is Mind Design, Mind Design II. MIT Press, Cambridge, MA

15. Van der Hoek W, Wooldridge M (2003) Towards a logic of rational agency. Log J IGPL 11(2):133-157

16. Bratman ME (1987) Intention, Plans, and Practical Reason. Harvard University Press, Cambridge, MA

17. Garbis C, Artman H (1998) Coordination and Communication as Distributed Cognition. In: Darses F, Zarate P (eds) Proceedings of Conference on the Design of Cooperative Systems (COOP'98). Cannes, France, pp 1-12

18. Cambria E, Schuller B, Xia YQ, Havasi C (2013) New avenues in opinion mining and sentiment analysis. IEEE Intell Syst 28(2):15-21

19. Ashby WR (1960) Design for a brain: The origin of adaptive behavior, Second Editionth edn. John Wiley \& Sons Inc, New York

20. Ashby WR (1958) Requisite variety and implications for control of complex systems. Cybernetica 1(2):83-99

21. Wiener N (1948) Cybernetics or Control and Communication in the Animal and Machine. MIT Press, Cambridge, MA

22. Albus J, Meystel A (2001) Engineering of Mind: An introduction to the science of intelligent systems. John Wiley Inc., New York, NY

23. Ashby WR (1956) An Introduction to Cybernetics. Chapman and Hall, London

24. Cambria E, Song Y, Wang H, Howard N (2013) Semantic multi-dimensional scaling for open-domain sentiment analysis. IEEE Intell Syst. doi:10.1109/MIS.2012.118

25. Cambria E, Mazzocco T, Hussain A (2013) Application of multi-dimensional scaling and artificial neural networks for biologically inspired opinion mining. Biologically Inspired Cognitive Architectures 4:41-53

26. Krippendorff K (1986) A Dictionary of Cybernetics. The American Society for Cybernetics, Norfolk VA

27. Maturana H, Varela FJ (1980) Autopoiesis and Cognition: The realization of the living, Volume 42nd edn. D. Riedel Publishing Company, Dordrecht, Holland

28. Varela FJ, Maturana H, Uribe R (1974) Autopoiesis: The organization of living systems, its characterization and a model. Biosystems 5(4):187-196

29. Ruiz-Mirazo K, Moreno A (2004) Basic autonomy as a fundamental step in the synthesis of life. Artif Life 10(3):235-259

30. Wooldridge M (2000) Reasoning about Rational Agents. The MIT Press, Cambridge, MA

31. Bruner JS (1981) Intention in the structure of action and interaction. In: Lewis L (ed) Advances in Infancy Research. Ablex, New Jersey

32. Epstein S (2003) Cognitive-experiential self-theory of personality. In: Millon T, Lerner M (eds) Comprehensive Handbook of Psychology, vol 5. Wiley \& Sons, Hoboken, NJ, pp 159-184 
33. Cambria E, Olsher D, Kwok K (2012) Sentic Activation: A Two-Level Affective Common Sense Reasoning Framework, Proceedings of Twenty-Sixth Conference on Artificial Intelligence. AAAl, Toronto, Ontario, Canada, pp 186-192

34. Iyengar SS, Kamenica E (2007) Choice Overload and Simplicity Seeking. Center for Behavioral and Decision Research, Seminar Paper, Waterloo, Canada

35. Cambria E, Olsher D, Kwok K (2012) Sentic Panalogy: Swapping Affective Common Sense Reasoning Strategies and Foci. Proceedings of CogSci 2012 Conference, Sapporo, Japan, pp 174-179

doi:10.1186/2192-1962-3-9

Cite this article as: Howard and Cambria: Intention awareness: improving upon situation awareness in humancentric environments. Human-centric Computing and Information Sciences 2013 3:9.

\section{Submit your next manuscript to BioMed Central} and take full advantage of:

- Convenient online submission

- Thorough peer review

- No space constraints or color figure charges

- Immediate publication on acceptance

- Inclusion in PubMed, CAS, Scopus and Google Scholar

- Research which is freely available for redistribution

Submit your manuscript at www.biomedcentral.com/submit 